of the radiation, that is, the relative magnitudes of the direct and diffuse components or of the ratio $D_{h} / E_{h}$. If it is assumed that for a given locality and season of the year the quality of the radiation is predominantly a function of the solar altitude $a$, then this latter factor is a convenient parameter on which to base a system of characteristic lines for a given value of $\theta_{H}$. Typical characteristic lines are shown for $\theta_{H}=90^{\circ}$ and $\theta_{H}=40^{\circ}$ in Fig. 2. An analysis of the theory shows that if the diffuse component of radiation were uniformly distributed the slope $M$ of the characteristic lines would be $\left(1-D_{h} / E_{h}\right)$, but as the distribution is not uniform $\left(1-D_{h} / E_{h}\right)$ is slightly smaller than $M$. The total radiation on a steeply inclined surface also includes some radiation from the heated surface of the ground, but this ceases to be appreciable when the angle of inclination $\theta_{H}$ is below $60^{\circ}$.

It is intended to publish charts or tabulated values of the constants $C$ and $M$ for various solar altitudes throughout the yearly cycle in the London area, which will enable the values of $E_{i} / E_{h}$ to be calculated for any surface. The primary object of this communication, however, is to promote interest in the research in other countries and to stimulate the determination of similar data which will extend knowledge of the availability of solar radiation.

The Woolwich Polytechnic,

HAROLD HEYWOOD London, S.E.18.

'Robinson, N., and Rechnitzer, D., Coopération Mediterranéenne pour l'Energie Solaire, No. 4, 18 (1963).

'Kandratyev, K. J., and Manolova, M. P., Solar Energy, 4, No. 1, 14 $(1960)$.

\section{Electron Temperature in a Laser-heated Plasma}

WheN a high-power laser beam is focused on to a material target, a small inertially confined plasma is generated near the target surface. Theoretical work by Basov and Krokhin 1,2, verified by Dawson ${ }^{3}$, suggests that very hot plasmas $\left(\sim 10^{7} \mathrm{~K}\right)$ can ultimately be produced in this way. A spectroscopic investigation of plasmas generated by a small $Q$-switched ruby laser has already been reported. Further experiments have now been carried out using a larger $Q$-switched neodymium glass laser, and an estimate of the electron temperature attained in a carbon plasma is reported in this communication. Other results will be described in detail elsewhere.

The peak power density at the focus of the neodymium glass laser beam was about $10^{11} \mathrm{~W} \mathrm{~cm}^{-2}$. The output wave-length was 10,600 A. Each laser pulse lasted about $1 \mu \mathrm{sec}$ and consisted of three spikes. The energy in a pulse was about 1 joule. The focused beam broke down atmospheric air, so the target was placed in a vacuum. The plasma formed by the laser beam was imaged on the entrance slit of a Hilger medium spectrograph. When a graphite target was used, a single laser pulse was sufficient to produce a spectrogram on which lines of the $C I$, II, III, IV and V spectra were identified.

Kaufman and Williams ${ }^{5,6}$ have pointed out that under certain conditions the specific intensities of lines from atoms of the $\mathrm{HeI}$ isoelectronic sequence give a useful indication of the plasma electron temperature even when thermodynamic equilibrium is absent and the electron and ion densities are only very approximately known. A number of assumptions must be made whose validity will be discussed in a later paper. In particular, a Max. wellian distribution of electron velocities is assumed. The expression given by these authors for the specific intensity, $I$, divided by the product of the number densities of electrons, $n_{e}$, and of quadruply ionized carbon atoms in the ground state, $n_{i}$, has been evaluated for the observed $C V$ transition $2 s^{3} S_{1}-2 p^{3} P_{2}$ at $2270.91 \AA$ and is plotted as a function of the electron temperature, $T_{e}$, in Fig. 1 .

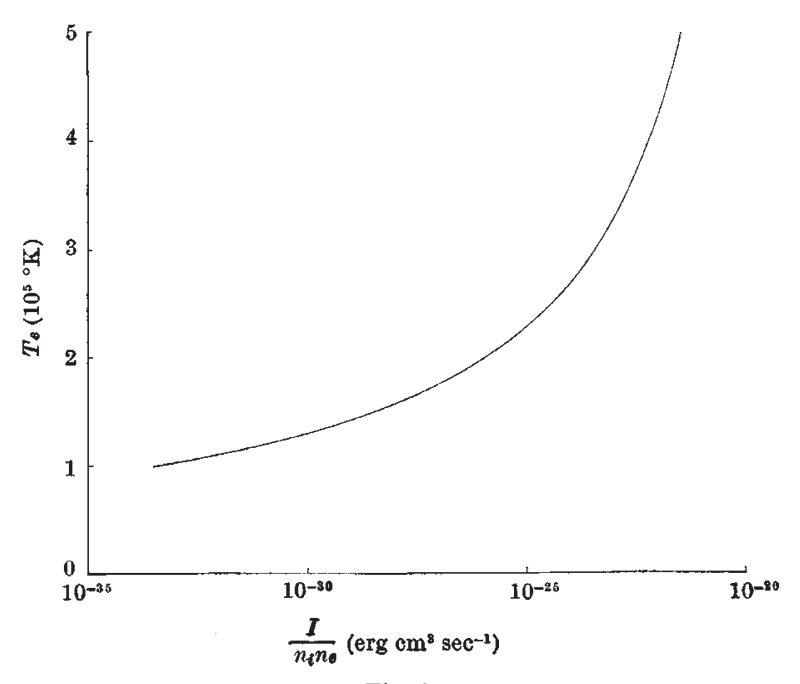

Fig. 1

Taking into account geometrical considerations, the observed blackening of the spectrographic plate indicates that the specific intensity of this $C \mathrm{~V}$ line was at least

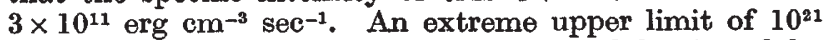
$\mathrm{cm}^{-3}$ for $n_{i}$ is obtained from the volume and density of the graphite removed from the target by the laser pulse, and the volume of luminous plasma emitting $C V$ light. The corresponding maximum electron density is $6 \times 10^{21}$ $\mathrm{cm}^{-3}$, because the carbon plasma was very pure. Thus $I /\left(n_{i} n_{e}\right) \geq 5 \times 10^{-82} \mathrm{erg} \mathrm{\textrm {cm } ^ { 3 }} \mathrm{sec}^{-1}$. From Fig. 1, the lower limit for the peak electron temperature is therefore $1 \cdot 1 \times 10^{5}{ }^{\circ} \mathrm{K}$. As may be seen from Fig. 1, at this temperature large errors in density or intensity estimates produce only small errors in the derived temperature. Nevertheless, as the true densities may well be orders of magnitude lower than the values used in the calculation the true electron temperature is probably somewhat higher.

E. Archbold

T. P. HUGHES

Light Division,

National Physical Laboratory, Teddington, Middlesex.

1 Basov, N. G., and Krokhin, O. N., Proc. Third Intern. Conf. Quantum llectronics, -1374 (Columbia, New York, 1964).

${ }^{2}$ Basov, N. G., and Krokhin, O. N., Soviet Physics, J.E.T.P., 19, 123 (1964). s Dawson, J. M., Phys. Fluids, 7, 981 (1964).

A Archbold, E., Harper, D. W., and Hughes, T. P., Brit. J. App. Phys., 15, 1321 (1964).

s Kaufman, S., and Williams, R. V., Nature,182, 557 (1958).

- Williams, R. V., and Kaufman, S., Proc. Phys. Soc., 75, 329 (1960).

\section{Saturation Characteristics of a Spherical Ionization Chamber and a Determination of Boag's Constant for Air}

Thomson and Rutherford' $\mathbf{s}^{1}$ initial investigations of the saturation characteristics of a parallel plate ionization chamber were followed by theoretical treatments by Thomson $^{2}, \mathrm{Mie}^{3}$, Seeliger ${ }^{4}$ and others. More recently, Boag and Wilson ${ }^{5}$ have shown by dimensional analysis that the collection efficiency, $f$ (that is, the ratio of the measured current, $i$, to the ideal saturation current, $\left.i_{8}\right)$, is a function of $d^{2} q^{\frac{1}{2}} V^{-1}$ where $d$ is the distance between the plates, $q$ is the rate of ionization and $V$ is the applied voltage. Through the introduction of certain assumptions, they derived the following equation for the generalized saturation characteristic:

$$
i_{s}=i\left[1+\frac{i}{i_{s}} \cdot \frac{1}{6} \frac{\alpha / e}{k_{1} k_{2}} \cdot \frac{d^{4} q}{V^{2}}\right]
$$

